SHORT REPORT

\title{
Paediatricians' perception of the use of extracorporeal membrane oxygenation to treat meconium aspiration syndrome
}

\author{
G M Walker, J A P Coutts, C Skeoch, C F Davis
}

Arch Dis Child Fetal Neonatal Ed 2003;88:F70-F71

This questionnaire study was designed to determine how consultant paediatricians perceived the value of extracorporeal membrane oxygenation (ECMO) in the treatment of meconium aspiration syndrome (MAS). A significant number believed that the survival of infants with MAS who received ECMO was lower than reported. Early discussion with an ECMO centre will ensure appropriate timing of referral.

A though there are guidelines aimed at preventing meconium aspiration syndrome (MAS), there are none governing the management of the sick neonate with established MAS. Some develop refractory persistent pulmonary hypertension of the newborn and hypoxia. Extracorporeal membrane oxygenation (ECMO) has been shown to increase survival without serious handicap in these infants. ${ }^{1}$ The purpose of this study was to determine consultant paediatricians' perception of the value of ECMO in the treatment of MAS.

\section{METHODS}

A questionnaire was designed to ascertain what treatments were considered appropriate before considering ECMO referral, timing and indications for referral, and the perceived mortality for infants with MAS treated with ECMO.

The Scottish Neonatal Consultants Group provided a database of consultant paediatricians involved in neonatal care in Scotland. A questionnaire was sent to each consultant. The four consultant neonatologists directly involved in the ECMO programme in Glasgow were excluded.

Returned questionnaires were scanned anonymously and read by optical character recognition software (FORMIC).

\section{RESULTS}

Thirty five of the 50 questionnaires were returned (70\%). Sixteen replies were from consultants with more than six sessions devoted to neonatology, and 19 from consultant paediatricians who provided neonatal cover. Fifteen respondents $(43 \%)$ worked in units where inhaled nitric oxide (iNO) was available.

\section{Treatment of MAS before considering ECMO referral}

Thirteen consultants (37\%) would use a systemic vasodilator, and $31 \%$ advocated hyperventilation to hypocarbia (table 1). Most respondents advocated a trial of iNO and high frequency oscillation ventilation. All who had iNO available would use it before referral, and $65 \%$ without access to iNO believed it should be used before referral.
Table 1 Treatments advocated before considering referral for extracorporeal membrane oxygenation

\begin{tabular}{lr}
\hline Treatment & $\mathrm{n}(\%)$ \\
\hline High frequency oscillation & $27(77)$ \\
Inhaled nitric oxide & $28(80)$ \\
Intravenous prostacyclin & $11(31)$ \\
Intravenous tolazoline & $6(17)$ \\
Intravenous magnesium & $6(17)$ \\
Hyperventilation to hypocarbia & $11(31)$ \\
\hline
\end{tabular}

\section{Indication for ECMO referral}

An oxygenation index (OI) greater than 20 would be used as an indicator for referral by $19 \%$ of respondents, an OI $>40$ by $22 \%$, and $47 \%$ would refer if there was no clinical improvement regardless of the OI.

\section{Time to ECMO referral}

Faced with the scenario of a deteriorating neonate with MAS, all respondents would refer before three days. However, given a scenario in which a ventilated neonate was showing no signs of improvement, one third would delay referral beyond three days.

\section{Predicted survival for neonate with MAS receiving ECMO}

Fourteen consultants (40\%) believed survival for a neonate with MAS on ECMO was about $90 \%$. Seventeen (49\%) believed survival was $60-80 \%$. Four respondents did not answer this question.

\section{DISCUSSION}

Neonates with severe MAS are ideal candidates for ECMO support. ${ }^{2}$ Most are near term and of sufficient weight to consider ECMO support. ECMO ensures adequate oxygenation and provides time for the pulmonary vasculature and parenchyma to recover while avoiding the damaging effects of high pressure ventilation and non-selective vasodilators. Ventilation parameters can be dramatically reduced, and intensive chest physiotherapy clears the meconium. The UK collaborative ECMO trial, the infants in which had an OI $>40$, indicated that there was one extra survivor for every four infants receiving ECMO for MAS. ${ }^{1}$

Abbreviations: MAS, meconium aspiration syndrome; ECMO,

extracorporeal membrane oxygenation; iNO, inhaled nitric oxide; $\mathrm{OI}$, oxygenation index; VVDL ECMO, venovenous ECMO where the right internal jugular vein is cannulated with a double lumen cannula 
Most patients during the UK collaborative ECMO trial (1993-1995) were supported with venoarterial ECMO, in which cannulae are inserted into the right internal jugular vein and right common carotid artery. More recently, venovenous ECMO, where the right internal jugular vein is cannulated with a double lumen cannula (VVDL ECMO), has become the technique of choice for neonates in the United Kingdom, ${ }^{2}$ thus avoiding ligation of the carotid artery. Increasingly, VVDL ECMO cannulae are inserted percutaneously.

Many respondents advocated other treatments for persistent pulmonary hypertension of the newborn before referring for ECMO support. Most (80\%) felt that a trial of iNO was appropriate, although only $43 \%$ had access to it. Of those without access to iNO, $65 \%$ felt it an appropriate treatment option before ECMO referral. The West of Scotland neonatal transport service regularly transports patients receiving iNO, the only limitations being the inability to use fixed wing air transport because of Civil Aviation Authority regulations. Other treatments for persistent pulmonary hypertension of the newborn continue to be advocated despite well documented side effects. Intravenous vasodilators have been shown to reduce systemic blood pressure..$^{3-5}$ Hyperventilation to hypocarbia contributes to pulmonary morbidity and reduces cardiac output. ${ }^{6}$

In a separate unpublished audit, we found that more than 72 hours of ventilation before referral was associated with longer ECMO runs. All respondents would refer a neonate who was deteriorating before three days, but, in a ventilated neonate with MAS who was not improving, one third would wait longer than three days before considering referral for ECMO. Over one fifth would not refer until the OI exceeds 40. A possible reason for the reluctance to refer early lies in the perception of the outcome of ECMO support. Nearly half the consultants believed that the survival of neonates with MAS who received ECMO was $80 \%$ or less. The Extracorporeal Life Support Organisation reports a worldwide survival rate of $94 \%$ for this group (personal communication). These data are considerably better than the $81 \%$ survival reported in the UK collaborative ECMO trial. Respondents who predicted poor survival may have based their predictions on this trial, entry to which required an $\mathrm{OI}>40$.
It is vital that paediatricians involved in the care of neonates with MAS are aware of the excellent results of ECMO support. Questionnaire results have been returned to all the Scottish neonatal consultants. Although we do not advocate ECMO support as the first line treatment for MAS, early contact with an ECMO centre will ensure optimal referral and the avoidance of unproven and often harmful treatments. Consideration of ECMO support before an OI of 40 is reached is supported by survival data, morbidity data, and increased use of non-invasive techniques such as percutaneous VVDL ECMO.

\section{ACKNOWLEDGEMENTS}

We thank Stephen Beaton for help in designing and reading the questionnaire. At the time of writing this article, GMW was supported by the SHERT/Cruden Medical Research Scholarship.

\section{Authors' affiliations}

G M Walker, J A P Coutts, C Skeoch, C F Davis, Department of Paediatric Surgery, Royal Hospital for Sick Children, Yorkhill Trust, Glasgow, Scotland, UK

Correspondence to: Dr Davis, Department of Paediatric Surgery, Royal Hospital for Sick Children, Yorkhill Trust, Glasgow G3 8SJ, Scotland, UK ; carl.davis@yorkhill.scot.nhs.uk

Accepted 20 July 2002

\section{REFERENCES}

1 UK Collaborative ECMO Group. UK collaborative randomised trial of neonatal extracorporeal membrane oxygenation. Lancet 1996:348:75-82.

2 Davis PJ, Shekerdemian LS. Meconium aspiration syndrome and extracorporeal membrane oxygenation. Arch Dis Child Fetal Neonatal Ed 2001;84:F1-3.

3 Bush A, Busst CM, Knight WB, et al. Comparison of the haemodynamic effects of epoprostenol (prostacyclin) and tolazoline. Br Heart J 1988;60:141-8

4 Kusuda S, Shishida N, Miyagi N, et al. Cerebral blood flow during treatment for pulmonary hypertension. Arch Dis Child Fetal Neonatal Ed 2000;80:F30-3.

5 Barrington KJ, Ryan CA, Finer NN. Effects of magnesium sulphate in a newborn piglet meconium aspiration model. J Perinatol 2000;20:373-8 6 Morris K, Beghetti M, Petros A, et al. Comparison of hyperventilation and inhaled nitric oxide for pulmonary hypertension after repair of congenital heart disease. Crit Care Med 2000;28:2974-8. 